\title{
Investigation of the photoelastic effect in Si at high values of the absorptivity
}

\author{
I. I. Boiko, Ye. F. Venger, E. V. Nikitenko, B. K. Serdega \\ Institute of Semiconductor Physics of NASU, 45, prospect Nauki, 252028 Kiev, Ukraine \\ Phone (044) 265-4020; e-mail: bshl@polarget.semicond.kiev.ua
}

\begin{abstract}
The birefrigence effect induced by uniaxial elastic deformation has been investigated in monocrystalline Si using theoretical and experimental technigues. To improve a measuring system sensitivity in the range of band absorption, polarization modulation of reffected emission was used. Deformation characteristics of this effect has been measured. The main results obtained for probing light wavelengths from the range of crystal transparency are in a good accordance with conclusions of previons studies. In the range of strong absorption, a considerable change of effect characteristic shapes was found, and their satisfactory accordance with results of theoretical estimations was also ascertained.
\end{abstract}

Keywords: birefrigence, polarization, modulation, photovoltage, anisotropy, thermal stress.

Paper received 10.03.99; revised manuscript received 01.06.99; accepted for publication 12.07.98.

1. The effect of birefringence in semiconducting crystals caused by uniaxial elastic deformation (also known as photoelastic or piezo-optical effect) has been investigated comprehensively and in much detail. This phenomenon, defined as the difference in the propagation velocity of waves polarized in two mutually orthogonal directions, is usually observed in anisotropic substances in the wavelength range corresponding to their transparency. It may seem that in materials with a strong absorption this effect can be observed only in an indirect way. For example, in [1] the components of the photoelastic tensor in silicon were measured by the Bragg diffraction technique, while in $[2,3]$ the photoelastic constants in the same material were calculated using Raman scattering measurements for several wavelengths from the fundamental absorption range and for the energy range 0.7 to $3.4 \mathrm{eV}$.

It is known that at large absorption coefficients the reflected wave is formed in the crystal layer of a thickness comparable to the absorption length. This indicates a finite length of the wave penetration into the crystal and a certain role of the bulk interaction. If the dielectric constant of the crystal is anisotropic, at least within the absorption length, the polarization of the reflected wave will be changed in comparison to the incident one. For example, a linearly polarized incident beam, upon reflection from the surface of such a crystal, in the general case becomes elliptical, if the polarization plane does not coincide with the axes of the optical indicatrix of the sample. Therefore, by detecting a change in the polarization of a normally incident beam reflected from the crystal surface, it is possible to observe the photoelastic effect directly.

However, in the range of fundamental absorption, the value of the polarization change, measured experimentally as the phase difference $\varphi$ between the orthogonal components of the wave, is by many orders of magnitude lower than that in the conditions of transparency. This is related to the fact that the length of the optical path appearing in the formula $\varphi \sim d \cdot \Delta n$ is now determined by the light absorption length rather than the thickness of the sample. Therefore, to observe this effect, one should use a technique having a high sensitivity with respect to the magnitude of the anisotropy. One of the techniques satisfying this requirement is based on the modulation of the probing beam polarization [4].

In the present paper, we study the birefringence in single crystals of $\mathrm{Si}$ in the fundamental absorption range. The anisotropy of the dielectric constant is induced by a uniaxial elastic deformation controlled by the external force.

2. Let us consider this phenomenon qualitatively. We assume that the dielectric properties of a semi-infinite crystal are determined by a diagonal tensor 


\section{I. Boiko et al.: Investigation of the photoelastic effect in Si at high...}

$\widehat{\varepsilon}=\left|\begin{array}{ccc}\varepsilon_{x} & 0 & 0 \\ 0 & \varepsilon_{y} & 0 \\ 0 & 0 & \varepsilon_{z}\end{array}\right|$

and the geometry of the experiment is such that the electric and magnetic components of the wave are presented by the vectors $\mathrm{E}=\left(E_{x}, E_{y}, 0\right), \mathrm{H}=\left(H_{x}, H_{y}, 0\right)$. In this case we assume $\nabla=\left(0,0, \nabla_{z}\right)$.

Then, Maxwell's equations

$$
\left\{\begin{array}{l}
\operatorname{rot} \mathrm{H}=\frac{1}{c} \widehat{\varepsilon} \cdot \frac{\partial \mathrm{E}}{\partial t} \\
\operatorname{rot} \mathrm{E}=-\frac{1}{c} \cdot \frac{\partial \mathrm{H}}{\partial t}
\end{array} \quad \nabla \times(\nabla \times \mathrm{E})=\frac{\omega^{2}}{c^{2}} \widehat{\varepsilon} \cdot \mathrm{E}\right.
$$

for waves $\mathrm{E}, \mathrm{H} \infty e^{-i \omega t+i k \mathrm{z}}$ are reduced to the equations $\left(k^{2}-\frac{\omega^{2}}{c^{2}} \varepsilon_{x}\right) E_{x}=0,\left(k^{2}-\frac{\omega^{2}}{c^{2}} \varepsilon_{y}\right) E_{y}=0$

Here, $k_{x}=\frac{\omega}{c} \sqrt{\varepsilon_{x}}=k_{0} \sqrt{\varepsilon_{x}}, k_{y}=$

$$
=k_{0} \sqrt{\varepsilon_{y}}, \varepsilon_{j}=\varepsilon_{j}^{\prime}+i \cdot \varepsilon_{j}^{\prime \prime} \quad(j=x, y) .
$$

The electric field and its gradient in the region $\mathrm{z} \leq 0$ :

$$
\begin{aligned}
& E_{j}=E_{1 j} \cdot e^{i k_{0} \cdot z}+E_{2 j} \cdot e^{-i k_{0} \cdot z} \\
& \nabla_{z} E_{j}=i k_{0} \cdot\left(E_{1 j} \cdot e^{i k_{0} \cdot z}-E_{2 j} \cdot e^{-i k_{0} \cdot z}\right)
\end{aligned}
$$

In the region $z \geq 0$

$$
\begin{aligned}
& E_{j}=E_{3 j} \cdot e^{i k \cdot z} \\
& \nabla_{z} E_{j}=E_{3 j} \cdot i k \cdot e^{i k \cdot z}
\end{aligned}
$$

Here $E_{1 j}$ is the amplitude of the incident wave, so that for $E_{2 j}$

$$
E_{1}+E_{2}=E_{3}, \quad i k\left(E_{1}-E_{2}\right)=i k \cdot E_{3}
$$

The amplitude of the reflected wave $E_{2 j}$ expressed through that of the incident wave $E_{1 j}$ is

$$
E_{1 j}+E_{2 j}=\frac{k_{0}}{k}\left(E_{1 j}-E_{2 j}\right)
$$

Thus, we obtain the two components of the wave:

$$
E_{2 x}=E_{1 x} \frac{1-\sqrt{\varepsilon_{x}}}{1+\sqrt{\varepsilon_{x}}}, \quad E_{2 y}=E_{1 y} \frac{1-\sqrt{\varepsilon_{y}}}{1+\sqrt{\varepsilon_{y}}}
$$

Let us separate the real and imaginary parts of the refractive index:

$\sqrt{\varepsilon_{x}}=n_{x}=n_{x}^{\prime}+i n_{x}^{\prime \prime}, \quad \sqrt{\varepsilon_{y}}=n_{y}=n_{y}^{\prime}+i n_{y}^{\prime \prime}$

Substitution of them in Eq. (7) yields the phase of the reflected wave:

$\delta=\operatorname{arctg}\left[\frac{\operatorname{Im} E_{2 x}}{\operatorname{Re} E_{2 x}}\right]-\operatorname{arctg}\left[\frac{\operatorname{Im} E_{2 y}}{\operatorname{Re} E_{2 y}}\right]$

Here $\operatorname{Re} E_{j}$ and $\operatorname{Im} E_{j}$ are the real and imaginary parts of the $j$-th component of the wave field.

Substituting (7) and (8) in (9), we obtain the final equation for the phase of the reflected wave:

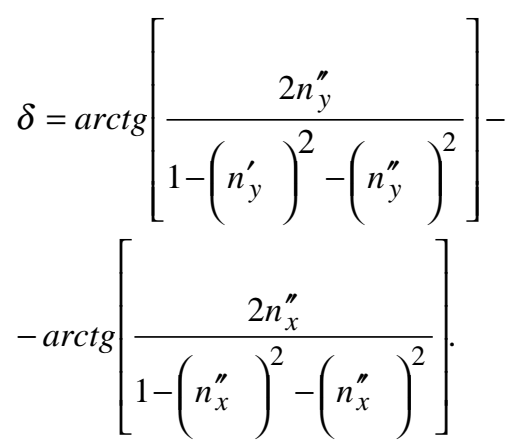

We assume the refractive indices to be linear functions of the uniaxial stress $X$. They can be expressed as

$$
\begin{aligned}
& n_{x}^{\prime}=n^{\prime}+c \cdot X, n_{x}^{\prime \prime}=n^{\prime \prime}+c \cdot X, n_{y}^{\prime}= \\
& =n^{\prime}-\gamma c X, n_{y}^{\prime \prime}=n^{\prime \prime}-\gamma c X .
\end{aligned}
$$

Here $\mathrm{c}$ is the photoelasticity constant for a given wavelength, and $\gamma$ is the Poisson coefficient.

Fig. 1 shows the dependence of the phase $\delta$ of the reflected wave on $c$ and $X$, which are the most significant factors. One can clearly see an anisotropy, which in the linear approximation is related to the uniaxial stress. The magnitude of the photoelasticity effect in the material under study is also affected by the Brewster constant $c$. As we might expect, in the isotropic case (at $X=0$ ) the polarization of light is determined only by the «metallic» character of reflection. This can be seen from the fact that every component of the wave changes its phase in the same manner. The difference of the wave phases appears only at $X \neq 0$ (when the anisotropy is induced), which was detected by our measurements.

3 . For experimental studies of the birefringence manifested in the reflected wave, we used a modified optical 


\section{I. Boiko et al.: Investigation of the photoelastic effect in Si at high...}

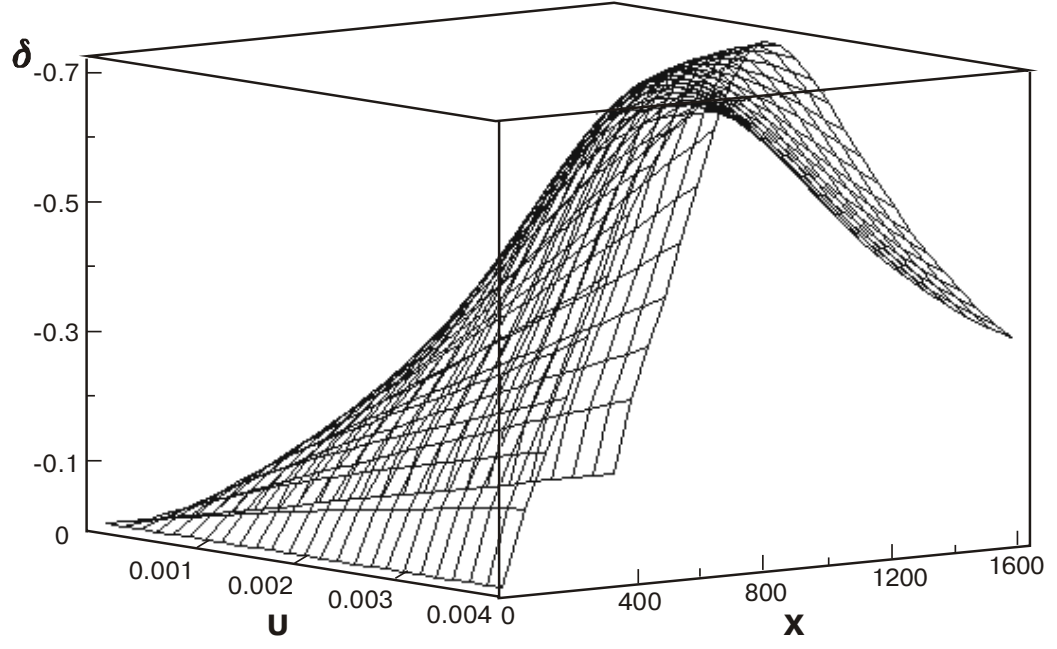

Fig. 1. A set of dependencies of the phase difference calculated by Eq.(10) for two orthogonal components of the polarized light in the presence of an anisotropy created by a mechanical stress $X$. The values used for calculations are: $n=3.8 ; c=0.04 ; g=0.3$.

polarization technique, in which the phase difference $\varphi$ between the orthogonal components of the wave was measured after passing through a sample with the thickness $d$ and anisotropy $\Delta n=n_{\perp}-n_{\mathrm{II}}$. Here $n_{\perp}, n_{\mathrm{II}}$ are the refractive indices in the directions of the optical indicatrix axes, and $\varphi=2 \pi \Delta n d / \lambda$. We had to elaborate this technique, since the experimental condition required a higher sensitivity with respect to the magnitude of $\Delta n$. Indeed, in the case of a strong absorption the length of the optical path is no longer determined by the crystal thickness $d$, as in the transparency case, but takes the values comparable to the absorption length, which is much less than $d$.

The elaboration of the technique consisted in using a polarization modulator, and in a modification of the optical scheme of measurements, so that the polarization state was analyzed and recorded in comparison to the reference signal [4]. The probe beam in this case was directed normally to the sample surface, in order to avoid the Fresnel distortion of the reflected beam. As a result, the detection capability of the technique with respect to birefringence was improved to the level of $\Delta \varphi=2 \pi \times 10^{-6}$ (in terms of phase), which is approximately equivalent to one angular second.

The magnitude of the birefringence was measured in the samples of single-crystalline silicon. The optical anisotropy was induced by a uniaxial elastic deformation induced by an external force applied in parallel to the illuminated surface. Rectangular samples with dimensions $10 \times 5 \times 3 \mathrm{~mm}^{3}$ were cut from a lightly doped $(\rho=200 \mathrm{Ohm} \cdot \mathrm{cm}) n$-type single-crystal ingot in such a way that the faces were parallel to the crystallographic planes $\{100\}$. The surfaces of the samples illuminated by the probing light were subjected to two kinds of treatment: mechanical and chemical mirror polishing.

During the sample preparation, a special attention was paid to the treatment of their faces to which the compression forces were applied. Their surfaces should be not only parallel to each other, but also geometrically flat. This is necessary both to ensure the uniformity of deformation over the cross-section of the sample and to eliminate any bending, which could cause a deflection of the reflected beam during the process of deformation. Furthermore, the deforming system was equipped with an independent source of the compressive force, to ensure the immobility of the entire system with respect to the beam.

Since the orientation of the optical indicatrix of the sample deformed by the uniaxial stress is known (it is determined by the direction of stress), then, as shown by the analysis of the entire system based on the matrix optics approach [5], the optimal polarization of the incident beam is the linear one. In this case, the polarization analyser consisting of a polarization modulator and a linear polarizer was set (using the adjustment of the azimuthal angle) to record the circular polarization component transformed from the initially linear one due to the interaction of light with the sample. A germanium photodiode with an enlarged receiving area was used as a photodetector. Its signal $I$ in this case is characterized by the relation $I \approx \sin \Delta \cdot \sin \left(\delta_{0} \sin \omega \cdot t\right)$, where $\Delta$ is the birefringence magnitude in the sample; $\delta_{0}$ is the modulation depth of the polarization modulator expressed through the value of the ellipticity angle; $\omega$ is the main frequency of the modulator. Note that at $\Delta<1$ the magnitude of the circular component of the beam (proportional to the photodetector signal) is a measure of the sample anisotropy, i.e. $I \sim \Delta$. A He-Ne laser LG-126 was used as a source of probing light. Its radiation with the wavelength $I \sim \Delta$ is weakly absorbed in silicon crystals at room temperature; $\alpha \cong 1 \mathrm{~cm}^{-1}$ (see [6]). It was used to compare the results of the modulation technique in the transparency region with those of other authors. Radiation with the wavelength $\lambda=0.63$ is absorbed, according to [6], over the length of $2.25 \cdot 10^{-4}$, which corresponds, at the sample thickness of $3 \mathrm{~mm}$, to the condition of a semi-infinite media. The signal, amplified by a loc-in nanovoltmeter, was recorded by a plotter at various magnitudes of the mechanical stress in the sample. The magnitude of the stress was measured using a silicon transformer based 


\section{I. Boiko et al.: Investigation of the photoelastic effect in Si at high...}

on the transverse tenso-emf (see [7]), placed in the deforming unit in such a way that it was subjected to the same force. Taking into account the high degree of linearity of the transformer's characteristics, this technique of measurements provided reproducible and reliable results.

4. First of all, we should note that, at a chosen doping level of the samples, the experimental results obtained are attributed to a mechanism of birefringence related to the deformation of the crystal lattice. This is the fact that should be taken into account when discussing the results of the polarization-modulation technique shown in Fig. 2. The dependence of the photodetector signal on the magnitude of the compressive force is plotted for measurements at the wavelength $\lambda=1.15 \mu \mathrm{m}$. It generally agrees with the results of birefringence studies reported by other authors (see, for example, [8]).

Indeed, these oscillations of the signal, like those observed by other researchers, are related to a variation of the phase difference between the two orthogonal components by a magnitude multiple to $2 \pi$. (By the way, this formal attribute has motivated the introduction of the notion of fringe order into the mechanics of the elastic deformed body). At the same time, the curve shown in Fig. 2 displays such features as variation of the period and amplitude and the asymmetry of oscillations, which make it different from the similar dependencies reported in other papers. To understand these peculiarities, note that it was established, using this technique, that crystals free of any intrinsic stresses are not available in practice. Therefore, even without any external stress, the polarization of radiation is changed under the action of intrinsic stresses already present in the crystal, which results in the appearance of the initial signal. This is the factor allowing the magnitude of birefringence to be measured within the first period of oscillations, which is an advantage of our technique.

As for the variations of the period and amplitude, they can be explained by the same reason - namely, by sample nonuniformities which can be of at least two types. The first type of nonuniformities is the uncontrollable variation of the composition in the direction of light propagation and a nonuniform deformation of the crystal related to this. The

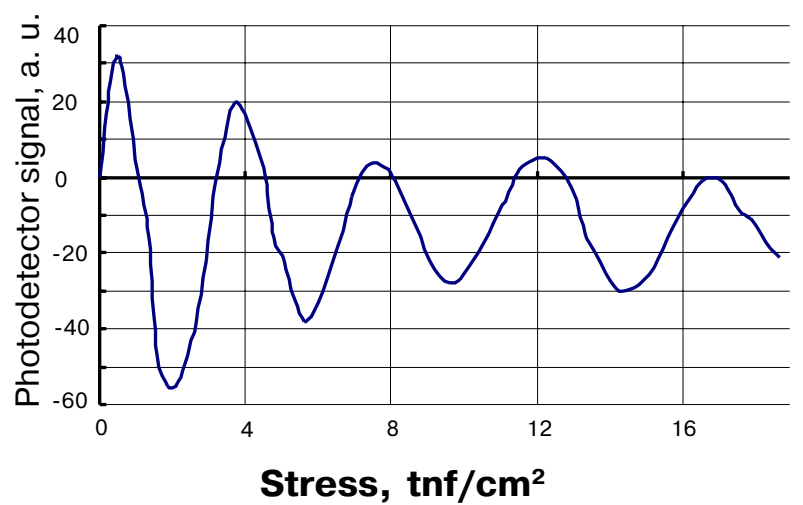

Fig. 2. An experimental dependence of the photodetector signal induced by radiation with the wavelength of $1.15 \mathrm{~mm}$ passed through a Si sample plotted as a function of the uniaxial stress. second type is related to a different value of the refractive index near the sample surface in comparison to that in the bulk. Its main peculiarity consists in the fact that the deformation characteristic of the photoelasticity effect in the surface layer can differ from that in the uniform bulk, and may even have the opposite sign.

The photoelasticity constant determined from Figure 1 is $\left(\varepsilon^{\perp}-\varepsilon^{\|}\right) / \varepsilon=6.8 \cdot 10^{-6}$, which in the units comparable to those employed in [8] differs only by $30 \%$ from the value reported there for the wavelength used in this paper.

When using the probing beam in the fundamental absorption range, the deformation characteristics of birefringence change dramatically. First of all, the signal becomes weaker by 3 to 4 orders of magnitude (at equal intensities of the incident radiation). Furthermore, the dependence of the phase of the reflected wave on the magnitude of the mechanical stress changes qualitatively. This curve, shown in Figure 3 by a thick line, is juxtaposed with that theoretically calculated according to Eq.(10) under the assumption of the linear dependence of the anisotropy on the stress $n_{x}-n_{y}=c$ $(1+\gamma) \cdot X$ (thin line). Since the calculations have not explicitly taken into account the dispersion of the refractivity, the above agreement between the theory and experiment can be considered as quite satisfactory. This is even more so in view of the fact that the photoelasticity constants in such a material as $\mathrm{Si}$, where the energy of vertical band transition is located far from the minimum bandgap, have a weak dispersion not only in the transparency range, but also in the case of a strong absorption of light. This could be easily verified by comparing the two practically identical deformation characteristics obtained in the reflection mode for the wavelengths of 1.15 and $0.63 \mu \mathrm{m}$.

It was unexpected to find that the phase dependences shown in Fig. 3 were nonmonotonic. This fact can be understood using analysis of Eq.(10) in which every orthogonal component of the wave consists, in its turn, of two terms. The first term is responsible for the surface reflection related to the presence of an interface between the two media, while the second one is attributed to the «metallic» reflection caused by a strong absorption. Each of these mechanisms in the case of an anisotropic tensor of the dielectric constant has a

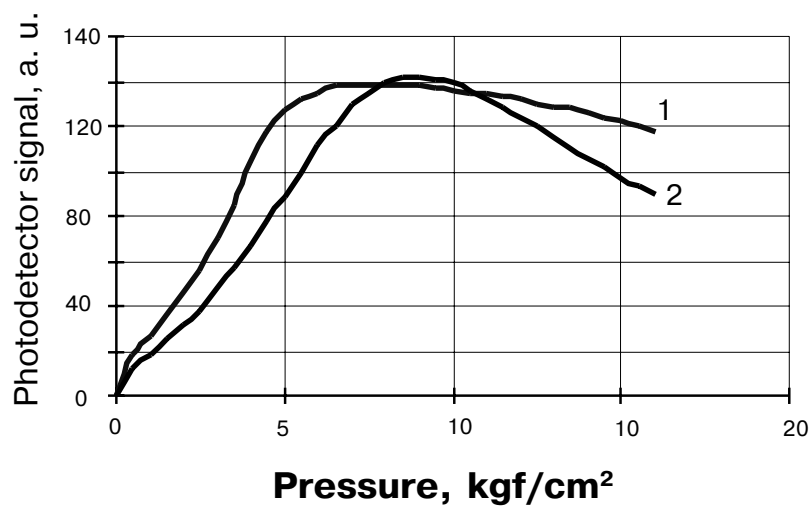

Fig. 3. The experimental (line 1) and calculated (line 2) dependences of the wave phase on the mechanical stress $X$. The theoretical curve is calculated using Eq.(10) for $n=3.8 ; g=0.3 ; c=0.001$. 


\section{I. Boiko et al.: Investigation of the photoelastic effect in Si at high...}

different effect on the phase magnitude, resulting in the appearance of the above-mentioned peculiarities.

The investigated photoelasticity effect manifested in the reflected beam could be used as a technique to measure the optical and mechanical parameters of the crystal. However, the presence of the features described above can be a strong factor hindering such measurements. Nevertheless, the considered effect is undoubtedly of great practical importance. It can be used both to determine the critical points of the band spectrum and to characterize the uniformity of semiconducting materials. Polarization analysis of the reflected radiation can provide information about the anisotropy of the material, caused either by the growth conditions or by an external action, for example, in the case of an elastic deformation of a cubic crystal. Since the presence of intrinsic mechanical stresses may be related to a gradient of the density of impurities or other defects of the crystal lattice, this technique is capable of both detecting and, if the calibration curve $\Delta=f(x)$ is known, assessing them quantitatively. Furthermore, the fact that the dispersion of the Brewster constant in the fundamental absorption range of $\mathrm{Si}$ is small makes it possible to detect a variation of the mechanical stress towards the crystal bulk by probing it with the light of a variable wavelength.

We have considered here only one case of orientation of the incident wave and the deforming force. Studies of other orientations offer additional possibilities. In that case the photoelasticity effect manifested in the reflected beam in the fundamental absorption range will be characterized by a tensor whose components may have, in the vicinity of the critical points of the Brillouin zone, an especially strong dispersion.

\section{References}

1. D. K. Biegelsen, Photoelastic Tensor of Silicon and the Volume Dependence of the Average Gap // Phys. Rev. Lett. 32 (21), p.1196 (1974).

2. M. Grimsditch, M. Cardona, Absolute Cross-Section for Raman Scattering by Phonons in Silicon // Phys. Stat. Sol. 102 (1), p.155 (1980).

3. M. Chandrasekar, M. Grimsditch, M. Cardona, Piezobirefringence above the fundamental edge in Si // Phys. Rev. B 18 (8), p.4301 (1978).

4. B. K. Serdega. Sposob izmereniya dvoinogo lucheprelomleniya (A technique of birefringence measurements), Ukrainian Patent No. 19983A of 01.07.94 (in Russian).

5. A. Gerard, C. M. Burch. Vvedenie v matrichnuyu optiku (Introduction to matrix optics).- Moscow, Mir Publ., 1978 (Russian translation)

6. W. C. Dash, R. Newman, Intrinsic Optical Absorption in SingleCrystal Ge and Si at $77 \mathrm{~K}$ and $300 \mathrm{~K} / /$ Phys. Rev. 99 (4), p.11511155 (1955).

7. I. P. Zhad'ko, V. A. Romanov, B. K. Serdega // Pribory i tekhnika eksperimenta, No.5, pp.1151-1155 (1972) (in Russian).

8. C. W. Higgingbotham, M. Cardona, F. H. Pollak, Intrinsic Piezobirefringence of Ge, Si, and GaAs // Phys. Rev. 184 (3), p.821-829 (1969). 